Identification and Structure-Activity Relationship Study of Imidazo[1,2-a]pyridine-3-amines $\quad-$ as First Selective Inhibitors of Excitatory Amino Acid Transporter Subtype 3 (EAAT3)

Peng $\mathrm{Wu}^{\dagger}$, Walden E. Bjørn-Yoshimoto ${ }^{\dagger}$, Markus Staudt, Anders A. Jensen* and Lennart Bunch*

Department of Drug Design and Pharmacology, Faculty of Health and Medical Sciences, University of Copenhagen, Copenhagen DK-2100, Denmark

$\dagger$ Co-first authors, contributed equally to this work.

*Corresponding authors: AAJ (pharmacology), E-mail: aaj@sund.ku.dk, LB (chemistry), Email: 1ebu@sund.ku.dk 
Table of content

Page S3-S14: 1H NMR and LCMS data for compounds 3b,d,g,i-n,o-z; 4b,d-f; 5d-f; 6c; 8a-d; 10a-g 


\section{Compound 3b}<smiles>Cc1ccccc1Nc1c(-c2ccco2)nc2ccccn12</smiles>

${ }^{1} \mathrm{H}$ NMR $\left(400 \mathrm{MHz}, \mathrm{CDCl}_{3}\right) \delta 7.78(\mathrm{~d}, J=6.8 \mathrm{~Hz}, 1 \mathrm{H}), 7.63(\mathrm{~d}, J=9.1 \mathrm{~Hz}, 1 \mathrm{H}), 7.44(\mathrm{~d}, J=$ $1.8 \mathrm{~Hz}, 1 \mathrm{H}), 7.25-7.17(\mathrm{~m}, 2 \mathrm{H}), 6.95(\mathrm{t}, J=7.5 \mathrm{~Hz}, 1 \mathrm{H}), 6.89-6.70(\mathrm{~m}, 3 \mathrm{H}), 6.44(\mathrm{dd}, J=$ $3.4,1.8 \mathrm{~Hz}, 1 \mathrm{H}), 6.08(\mathrm{~d}, J=8.0 \mathrm{~Hz}, 1 \mathrm{H}), 5.67$ (s, 1H), 2.46 (s, 3H). MS (ESI+) $m / z: 290.0$ $[\mathrm{M}+\mathrm{H}]^{+}$.

\section{Compound 3d}<smiles>Cc1ccc2nc(-c3ccco3)c(Nc3ccccc3C)n2c1</smiles>

${ }^{1} \mathrm{H}$ NMR $\left(400 \mathrm{MHz}, \mathrm{CDCl}_{3}\right) \delta 7.66-7.57(\mathrm{~m}, 1 \mathrm{H}), 7.54(\mathrm{~d}, J=9.1 \mathrm{~Hz}, 1 \mathrm{H}), 7.48-7.39(\mathrm{~m}$, $1 \mathrm{H}), 7.21(\mathrm{~d}, J=7.4 \mathrm{~Hz}, 1 \mathrm{H}), 7.08(\mathrm{dd}, J=9.2,1.8 \mathrm{~Hz}, 1 \mathrm{H}), 7.02-6.92(\mathrm{~m}, 1 \mathrm{H}), 6.88-6.77$ $(\mathrm{m}, 1 \mathrm{H}), 6.76-6.65(\mathrm{~m}, 1 \mathrm{H}), 6.42(\mathrm{dd}, J=3.4,1.8 \mathrm{~Hz}, 1 \mathrm{H}), 6.09(\mathrm{~d}, J=9.5 \mathrm{~Hz}, 1 \mathrm{H}), 5.63(\mathrm{~s}$, $1 \mathrm{H}), 2.47(\mathrm{~s}, 3 \mathrm{H}), 2.28(\mathrm{~s}, 3 \mathrm{H})$. MS (ESI+) $\mathrm{m} / z: 304.1[\mathrm{M}+\mathrm{H}]^{+}$.

\section{Compound 3g}<smiles>Cc1ccccc1Nc1c(-c2ccco2)nc2ccc(Br)cn12</smiles>

${ }^{1} \mathrm{H}$ NMR $\left(600 \mathrm{MHz}, \mathrm{CDCl}_{3}\right) \delta 7.99(\mathrm{~s}, 1 \mathrm{H}), 7.56(\mathrm{~d}, J=9.4 \mathrm{~Hz}, 1 \mathrm{H}), 7.47-7.42(\mathrm{~m}, 1 \mathrm{H})$, $7.30(\mathrm{dd}, J=9.5,1.9 \mathrm{~Hz}, 1 \mathrm{H}), 7.23(\mathrm{~d}, J=7.5 \mathrm{~Hz}, 1 \mathrm{H}), 7.01-6.95(\mathrm{~m}, 1 \mathrm{H}), 6.87-6.81(\mathrm{~m}$, $1 \mathrm{H}), 6.74(\mathrm{~d}, J=3.4 \mathrm{~Hz}, 1 \mathrm{H}), 6.44(\mathrm{dd}, J=3.4,1.8 \mathrm{~Hz}, 1 \mathrm{H}), 6.07(\mathrm{~d}, J=8.1 \mathrm{~Hz}, 1 \mathrm{H}), 5.86(\mathrm{~s}$, 1H), 2.49 (s, 3H). MS (ESI+) m/z: $368.1\left[\mathrm{M}+\mathrm{H},{ }^{79} \mathrm{Br}\right]^{+}, 370.1\left[\mathrm{M}+\mathrm{H},{ }^{81} \mathrm{Br}\right]^{+}$.

\section{Compound 3i}


<smiles>Cc1ccccc1Nc1c(-c2ccco2)nc2c(C(F)(F)F)cccn12</smiles>

${ }^{1} \mathrm{H}$ NMR $\left(400 \mathrm{MHz}, \mathrm{CDCl}_{3}\right) \delta 7.87(\mathrm{~d}, J=6.8 \mathrm{~Hz}, 1 \mathrm{H}), 7.53(\mathrm{~d}, J=7.1 \mathrm{~Hz}, 1 \mathrm{H}), 7.45(\mathrm{~s}, 1 \mathrm{H})$, $7.28-7.19(\mathrm{~m}, 1 \mathrm{H}), 6.95(\mathrm{t}, J=7.7 \mathrm{~Hz}, 1 \mathrm{H}), 6.87-6.75(\mathrm{~m}, 3 \mathrm{H}), 6.45-6.39(\mathrm{~m}, 1 \mathrm{H}), 6.04$ $(\mathrm{d}, J=8.0 \mathrm{~Hz}, 1 \mathrm{H}), 5.66(\mathrm{~s}, 1 \mathrm{H}), 2.48(\mathrm{~s}, 3 \mathrm{H}) . \mathrm{MS}(\mathrm{ESI}+) \mathrm{m} / \mathrm{z}: 358.1[\mathrm{M}+\mathrm{H}]^{+}$.

\section{Compound 3j}<smiles>Cc1ccccc1Nc1c(-c2ccco2)nc2c(C#N)cccn12</smiles>

${ }^{1} \mathrm{H}$ NMR $\left(400 \mathrm{MHz}, \mathrm{CDCl}_{3}\right) \delta 7.93(\mathrm{~d}, J=7.0 \mathrm{~Hz}, 1 \mathrm{H}), 7.62(\mathrm{~d}, J=7.0 \mathrm{~Hz}, 1 \mathrm{H}), 7.46(\mathrm{~s}, 1 \mathrm{H})$, $7.23(\mathrm{~d}, J=7.4 \mathrm{~Hz}, 1 \mathrm{H}), 6.96(\mathrm{t}, J=7.6 \mathrm{~Hz}, 1 \mathrm{H}), 6.90-6.80(\mathrm{~m}, 3 \mathrm{H}), 6.47$ (dd, $J=3.5,1.8$ $\mathrm{Hz}, 1 \mathrm{H}), 6.01(\mathrm{~d}, J=8.0 \mathrm{~Hz}, 1 \mathrm{H}), 5.76(\mathrm{~s}, 1 \mathrm{H}), 2.48(\mathrm{~s}, 3 \mathrm{H}) . \mathrm{MS}(\mathrm{ESI}+) \mathrm{m} / z: 315.1[\mathrm{M}+\mathrm{H}]^{+}$.

\section{Compound 3k}<smiles>Cc1ccccc1Nc1c(-c2ccco2)nc2c(N)cccn12</smiles>

${ }^{1} \mathrm{H}$ NMR $\left(400 \mathrm{MHz}, \mathrm{CDCl}_{3}\right) \delta 7.44(\mathrm{dd}, J=1.8,0.7 \mathrm{~Hz}, 1 \mathrm{H}), 7.35(\mathrm{~d}, J=6.6 \mathrm{~Hz}, 1 \mathrm{H}), 7.20$ $(\mathrm{d}, J=7.3 \mathrm{~Hz}, 1 \mathrm{H}), 6.97(\mathrm{q}, J=7.4 \mathrm{~Hz}, 2 \mathrm{H}), 6.86-6.80(\mathrm{~m}, 1 \mathrm{H}), 6.76(\mathrm{~s}, 0 \mathrm{H}), 6.51(\mathrm{~d}, J=$ $7.6 \mathrm{~Hz}, 1 \mathrm{H}), 6.41(\mathrm{dd}, J=3.4,1.8 \mathrm{~Hz}, 1 \mathrm{H}), 6.11(\mathrm{~d}, J=8.0 \mathrm{~Hz}, 1 \mathrm{H}), 5.88(\mathrm{~d}, J=26.6 \mathrm{~Hz}$, $1 \mathrm{H}), 2.48(\mathrm{~s}, 3 \mathrm{H}) . m / z: 305.1[\mathrm{M}+\mathrm{H}]^{+}$.

\section{Compound 31}<smiles>Cc1ccccc1Nc1c(-c2ccco2)nc2c(C)cc(Cl)cn12</smiles> 
${ }^{1} \mathrm{H}$ NMR $\left(600 \mathrm{MHz}, \mathrm{CDCl}_{3}\right) \delta 7.66(\mathrm{~s}, 1 \mathrm{H}), 7.45(\mathrm{~s}, 1 \mathrm{H}), 7.20(\mathrm{~d}, J=7.4 \mathrm{~Hz}, 1 \mathrm{H}), 6.99-6.92$ $(\mathrm{m}, 2 \mathrm{H}), 6.81(\mathrm{t}, J=7.4 \mathrm{~Hz}, 1 \mathrm{H}), 6.60(\mathrm{~d}, J=3.4 \mathrm{~Hz}, 1 \mathrm{H}), 6.42-6.38(\mathrm{~m}, 1 \mathrm{H}), 6.05(\mathrm{~d}, J=$ $8.0 \mathrm{~Hz}, 1 \mathrm{H}), 5.54(\mathrm{~s}, 1 \mathrm{H}), 2.66(\mathrm{~s}, 3 \mathrm{H}), 2.46(\mathrm{~s}, 3 \mathrm{H}) . \mathrm{MS}(\mathrm{ESI}+) \mathrm{m} / \mathrm{z}: 338.1\left[\mathrm{M}+\mathrm{H},{ }^{35} \mathrm{Cl}\right]^{+}$, $340.1\left[\mathrm{M}+\mathrm{H},{ }^{37} \mathrm{Cl}\right]^{+}$.

\section{Compound 3n}

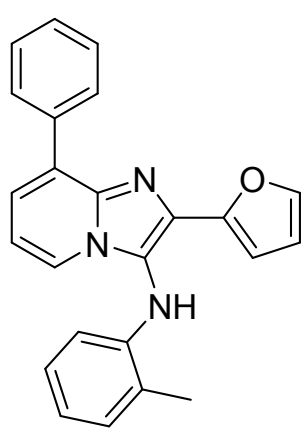

${ }^{1} \mathrm{H}$ NMR $\left(400 \mathrm{MHz}, \mathrm{CDCl}_{3}\right) \delta 8.12-8.04(\mathrm{~m}, 2 \mathrm{H}), 7.81(\mathrm{dd}, J=6.7,1.2 \mathrm{~Hz}, 1 \mathrm{H}), 7.57-$ $7.48(\mathrm{~m}, 2 \mathrm{H}), 7.46-7.41(\mathrm{~m}, 2 \mathrm{H}), 7.38(\mathrm{dd}, J=7.1,1.2 \mathrm{~Hz}, 1 \mathrm{H}), 7.22(\mathrm{~d}, J=7.4 \mathrm{~Hz}, 1 \mathrm{H})$, $6.98(\mathrm{ddd}, J=8.8,7.5,1.6 \mathrm{~Hz}, 1 \mathrm{H}), 6.90(\mathrm{t}, J=6.9 \mathrm{~Hz}, 1 \mathrm{H}), 6.88-6.78(\mathrm{~m}, 2 \mathrm{H}), 6.46-6.40$ (m, 1H), $6.14(\mathrm{~d}, J=7.9 \mathrm{~Hz}, 1 \mathrm{H}), 5.82(\mathrm{~s}, 1 \mathrm{H}), 2.48(\mathrm{~s}, 3 \mathrm{H}) . \mathrm{MS}(\mathrm{ESI}+) \mathrm{m} / z: 366.2[\mathrm{M}+\mathrm{H}]^{+}$.

\section{Compound 30}<smiles>Cc1ccccc1Nc1c(-c2ccco2)nc2cc(-c3ccccc3)ccn12</smiles>

${ }^{1} \mathrm{H}$ NMR $\left(400 \mathrm{MHz}, \mathrm{CDCl}_{3}\right) \delta 7.96-7.84(\mathrm{~m}, 2 \mathrm{H}), 7.64(\mathrm{~d}, J=8.5 \mathrm{~Hz}, 2 \mathrm{H}), 7.54-7.36(\mathrm{~m}$, 4H), $7.23-7.18(\mathrm{~m}, 1 \mathrm{H}), 7.16-7.06(\mathrm{~m}, 1 \mathrm{H}), 7.01-6.90(\mathrm{~m}, 1 \mathrm{H}), 6.88-6.75(\mathrm{~m}, 1 \mathrm{H}), 6.43$ $-6.30(\mathrm{~m}, 1 \mathrm{H}), 6.13(\mathrm{~d}, J=8.0 \mathrm{~Hz}, 1 \mathrm{H}), 6.08(\mathrm{~s}, 1 \mathrm{H}), 2.51(\mathrm{~s}, 3 \mathrm{H})$. MS (ESI+) $\mathrm{m} / z: 366.2[\mathrm{M}$ $+\mathrm{H}]^{+}$.

\section{Compound 3p}<smiles>COc1ccc(-c2cccn3c(Nc4ccccc4C)c(-c4ccco4)nc23)cc1</smiles> 
${ }^{1} \mathrm{H}$ NMR $\left(400 \mathrm{MHz}, \mathrm{CDCl}_{3}\right) \delta 8.16-8.01(\mathrm{~m}, 2 \mathrm{H}), 7.75(\mathrm{~d}, J=6.7 \mathrm{~Hz}, 1 \mathrm{H}), 7.44(\mathrm{~d}, J=1.8$ $\mathrm{Hz}, 1 \mathrm{H}), 7.32(\mathrm{~d}, J=7.1 \mathrm{~Hz}, 1 \mathrm{H}), 7.21(\mathrm{~d}, J=7.4 \mathrm{~Hz}, 1 \mathrm{H}), 7.11-7.01(\mathrm{~m}, 2 \mathrm{H}), 6.97(\mathrm{t}, J=$ $7.1 \mathrm{~Hz}, 1 \mathrm{H}), 6.90-6.79(\mathrm{~m}, 3 \mathrm{H}), 6.43(\mathrm{dd}, J=3.4,1.8 \mathrm{~Hz}, 1 \mathrm{H}), 6.14(\mathrm{dd}, J=8.1,1.2 \mathrm{~Hz}$, $1 \mathrm{H}), 5.74(\mathrm{~s}, 1 \mathrm{H}), 3.89(\mathrm{~s}, 3 \mathrm{H}), 2.47(\mathrm{~s}, 3 \mathrm{H})$.

\section{Compound 3q}<smiles>COc1ccc(-c2ccn3c(Nc4ccccc4C)c(-c4ccco4)nc3c2)cc1</smiles>

${ }^{1} \mathrm{H}$ NMR $\left(400 \mathrm{MHz}, \mathrm{CDCl}_{3}\right) \delta 7.74-7.68(\mathrm{~m}, 2 \mathrm{H}), 7.56-7.48(\mathrm{~m}, 2 \mathrm{H}), 7.39(\mathrm{dd}, J=1.8$, $0.7 \mathrm{~Hz}, 1 \mathrm{H}), 7.15(\mathrm{~d}, J=7.4 \mathrm{~Hz}, 1 \mathrm{H}), 7.01-6.92(\mathrm{~m}, 3 \mathrm{H}), 6.92-6.85(\mathrm{~m}, 1 \mathrm{H}), 6.75(\mathrm{td}, J=$ 7.4, 1.2 Hz, 1H), 6.67 (d, $J=3.4 \mathrm{~Hz}, 1 \mathrm{H}), 6.39$ (dd, $J=3.4,1.8 \mathrm{~Hz}, 1 \mathrm{H}), 6.09$ (dd, $J=8.1$, $1.1 \mathrm{~Hz}, 1 \mathrm{H}), 5.59(\mathrm{~s}, 1 \mathrm{H}), 3.80(\mathrm{~s}, 3 \mathrm{H}), 2.40(\mathrm{~s}, 3 \mathrm{H})$.

\section{Compound 3r}<smiles>COc1ccc(-c2ccc3nc(-c4ccco4)c(Nc4ccccc4C)n3c2)cc1</smiles>

${ }^{1} \mathrm{H}$ NMR $\left(400 \mathrm{MHz}, \mathrm{CDCl}_{3}\right) \delta 7.98-7.88(\mathrm{~m}, 1 \mathrm{H}), 7.68(\mathrm{dd}, J=9.3,1.0 \mathrm{~Hz}, 1 \mathrm{H}), 7.52-$ $7.38(\mathrm{~m}, 4 \mathrm{H}), 7.21(\mathrm{~d}, J=6.9 \mathrm{~Hz}, 1 \mathrm{H}), 7.09-6.86(\mathrm{~m}, 4 \mathrm{H}), 6.81(\mathrm{td}, J=7.4,1.3 \mathrm{~Hz}, 1 \mathrm{H})$, $6.72(\mathrm{~d}, J=3.4 \mathrm{~Hz}, 1 \mathrm{H}), 6.42(\mathrm{dd}, J=3.4,1.8 \mathrm{~Hz}, 1 \mathrm{H}), 6.14(\mathrm{~d}, J=8.1 \mathrm{~Hz}, 1 \mathrm{H}), 5.80(\mathrm{~s}, 1 \mathrm{H})$, $3.83(\mathrm{~s}, 3 \mathrm{H}), 2.49$ (s, 4H). MS (ESI+) $m / z: 396.3[\mathrm{M}+\mathrm{H}]^{+}$.

\section{Compound 3s}<smiles>COc1cc(F)ccc1-c1cccn2c(Nc3ccccc3C)c(-c3ccco3)nc12</smiles>

${ }^{1} \mathrm{H}$ NMR $\left(400 \mathrm{MHz}, \mathrm{CDCl}_{3}\right) \delta 8.01(\mathrm{dd}, J=8.4,2.1 \mathrm{~Hz}, 1 \mathrm{H}), 7.79(\mathrm{dd}, J=6.7,1.2 \mathrm{~Hz}, 1 \mathrm{H})$, $7.59-7.50(\mathrm{~m}, 1 \mathrm{H}), 7.44(\mathrm{dd}, J=1.8,0.8 \mathrm{~Hz}, 1 \mathrm{H}), 7.36(\mathrm{dd}, J=7.1,1.2 \mathrm{~Hz}, 1 \mathrm{H}), 7.26-7.15$ $(\mathrm{m}, 2 \mathrm{H}), 7.02-6.93(\mathrm{~m}, 1 \mathrm{H}), 6.93-6.78(\mathrm{~m}, 3 \mathrm{H}), 6.45(\mathrm{dd}, J=3.4,1.8 \mathrm{~Hz}, 1 \mathrm{H}), 6.13(\mathrm{~d}, J=$ $8.1 \mathrm{~Hz}, 1 \mathrm{H}), 5.76(\mathrm{~s}, 1 \mathrm{H}), 4.03(\mathrm{~s}, 3 \mathrm{H}), 2.48(\mathrm{~s}, 3 \mathrm{H}) . \mathrm{MS}(\mathrm{ESI}+) \mathrm{m} / z: 414.2[\mathrm{M}+\mathrm{H}]^{+}$. 


\section{Compound 3t}<smiles>COc1cc(F)ccc1-c1ccn2c(Nc3ccccc3C)c(-c3ccco3)nc2c1</smiles>

${ }^{1} \mathrm{H}$ NMR $\left(400 \mathrm{MHz}, \mathrm{CDCl}_{3}\right) \delta 7.85-7.76(\mathrm{~m}, 2 \mathrm{H}), 7.45(\mathrm{~d}, J=1.7 \mathrm{~Hz}, 1 \mathrm{H}), 7.24-7.19(\mathrm{~m}$, 2H), $7.19-7.10(\mathrm{~m}, 2 \mathrm{H}), 7.07-6.92(\mathrm{~m}, 2 \mathrm{H}), 6.87-6.75(\mathrm{~m}, 2 \mathrm{H}), 6.44(\mathrm{dd}, J=3.4,1.8 \mathrm{~Hz}$, $1 \mathrm{H}), 6.12(\mathrm{dd}, J=8.0,1.2 \mathrm{~Hz}, 1 \mathrm{H}), 5.80(\mathrm{~d}, J=3.0 \mathrm{~Hz}, 1 \mathrm{H}), 3.96(\mathrm{~s}, 3 \mathrm{H}), 2.48(\mathrm{~s}, 3 \mathrm{H})$.

\section{Compound 3u}<smiles>COc1cc(F)ccc1-c1ccc2nc(-c3ccco3)c(Nc3ccccc3C)n2c1</smiles>

${ }^{1} \mathrm{H}$ NMR $\left(400 \mathrm{MHz}, \mathrm{CDCl}_{3}\right) \delta 7.97(\mathrm{~s}, 1 \mathrm{H}), 7.73(\mathrm{~d}, J=9.3 \mathrm{~Hz}, 1 \mathrm{H}), 7.50-7.36(\mathrm{~m}, 2 \mathrm{H})$, $7.22(\mathrm{~d}, J=7.4 \mathrm{~Hz}, 1 \mathrm{H}), 7.17-7.04(\mathrm{~m}, 2 \mathrm{H}), 7.03-6.92(\mathrm{~m}, 2 \mathrm{H}), 6.82(\mathrm{t}, J=7.4 \mathrm{~Hz}, 1 \mathrm{H})$, $6.76(\mathrm{~d}, J=3.3 \mathrm{~Hz}, 1 \mathrm{H}), 6.42(\mathrm{dd}, J=3.5,1.7 \mathrm{~Hz}, 1 \mathrm{H}), 6.13(\mathrm{~d}, J=8.0 \mathrm{~Hz}, 1 \mathrm{H}), 6.06(\mathrm{~s}, 1 \mathrm{H})$, $3.92(\mathrm{~s}, 3 \mathrm{H}), 2.51(\mathrm{~s}, 3 \mathrm{H})$. MS (ESI+) $m / z: 414.2[\mathrm{M}+\mathrm{H}]^{+}$.

\section{Compound 3v}<smiles>COc1ccc(C#N)cc1-c1ccn2c(Nc3ccccc3C)c(-c3ccco3)nc2c1</smiles>

${ }^{1} \mathrm{H}$ NMR $\left(400 \mathrm{MHz}, \mathrm{CDCl}_{3}\right) \delta 7.84(\mathrm{~d}, J=7.4 \mathrm{~Hz}, 2 \mathrm{H}), 7.72-7.63(\mathrm{~m}, 2 \mathrm{H}), 7.45(\mathrm{~d}, J=1.8$ $\mathrm{Hz}, 1 \mathrm{H}), 7.23(\mathrm{~d}, J=7.4 \mathrm{~Hz}, 1 \mathrm{H}), 7.07(\mathrm{~d}, J=8.4 \mathrm{~Hz}, 1 \mathrm{H}), 6.98(\mathrm{td}, J=7.7,1.6 \mathrm{~Hz}, 2 \mathrm{H})$, $6.88-6.79(\mathrm{~m}, 2 \mathrm{H}), 6.44(\mathrm{dd}, J=3.4,1.8 \mathrm{~Hz}, 1 \mathrm{H}), 6.14(\mathrm{~d}, J=8.0 \mathrm{~Hz}, 1 \mathrm{H}), 5.84(\mathrm{~s}, 1 \mathrm{H})$, $3.93(\mathrm{~s}, 3 \mathrm{H}), 2.49$ (s, 3H). MS (ESI+) $m / z: 421.2[\mathrm{M}+\mathrm{H}]^{+}$.

\section{Compound 3w}<smiles>COc1ccc(C#N)cc1-c1ccc2nc(-c3ccco3)c(Nc3ccccc3C)n2c1</smiles> 
${ }^{1} \mathrm{H}$ NMR $\left(400 \mathrm{MHz}, \mathrm{CDCl}_{3}\right) \delta 7.98(\mathrm{~s}, 1 \mathrm{H}), 7.71(\mathrm{~d}, J=9.3 \mathrm{~Hz}, 1 \mathrm{H}), 7.64(\mathrm{dd}, J=8.7,2.2$ $\mathrm{Hz}, 1 \mathrm{H}), 7.58(\mathrm{~d}, J=2.1 \mathrm{~Hz}, 1 \mathrm{H}), 7.45(\mathrm{dd}, J=1.8,0.8 \mathrm{~Hz}, 1 \mathrm{H}), 7.38$ (dd, $J=9.3,1.8 \mathrm{~Hz}$, $1 \mathrm{H}), 7.21(\mathrm{~d}, J=6.9 \mathrm{~Hz}, 1 \mathrm{H}), 7.04-6.94(\mathrm{~m}, 2 \mathrm{H}), 6.87-6.78(\mathrm{~m}, 2 \mathrm{H}), 6.47-6.41(\mathrm{~m}, 1 \mathrm{H})$, $6.15(\mathrm{~d}, J=8.0 \mathrm{~Hz}, 1 \mathrm{H}), 5.90(\mathrm{~s}, 1 \mathrm{H}), 3.79(\mathrm{~s}, 3 \mathrm{H}), 2.49(\mathrm{~s}, 3 \mathrm{H})$. MS (ESI+) $\mathrm{m} / z: 421.2[\mathrm{M}+$ $\mathrm{H}]^{+}$.

\section{Compound 3x}<smiles>COc1ccc(-c2ccn3c(Nc4ccccc4C)c(-c4ccco4)nc3c2)c(CO)c1</smiles>

${ }^{1} \mathrm{H}$ NMR $\left(400 \mathrm{MHz}, \mathrm{CDCl}_{3}\right) \delta 7.83(\mathrm{dd}, J=7.0,0.9 \mathrm{~Hz}, 1 \mathrm{H}), 7.64-7.59(\mathrm{~m}, 1 \mathrm{H}), 7.44(\mathrm{dd}, J$ $=1.8,0.8 \mathrm{~Hz}, 1 \mathrm{H}), 7.25-7.20(\mathrm{~m}, 2 \mathrm{H}), 7.18(\mathrm{~d}, J=2.7 \mathrm{~Hz}, 1 \mathrm{H}), 7.02-6.88(\mathrm{~m}, 4 \mathrm{H}), 6.88-$ $6.81(\mathrm{~m}, 2 \mathrm{H}), 6.44(\mathrm{dd}, J=3.4,1.7 \mathrm{~Hz}, 1 \mathrm{H}), 6.16(\mathrm{dd}, J=8.0,1.2 \mathrm{~Hz}, 1 \mathrm{H}), 5.89(\mathrm{~s}, 1 \mathrm{H}), 4.68$ (s, 2H), $3.87(\mathrm{~s}, 3 \mathrm{H}), 2.49(\mathrm{~s}, 3 \mathrm{H})$. MS (ESI+) $\mathrm{m} / z$ : $426.2[\mathrm{M}+\mathrm{H}]^{+}$.

\section{Compound $3 y$}<smiles>COc1ccc(-c2ccc3nc(-c4ccco4)c(Nc4ccccc4C)n3c2)c(CO)c1</smiles>

${ }^{1} \mathrm{H}$ NMR (400 MHz, DMSO) $\delta 7.87-7.81(\mathrm{~m}, 1 \mathrm{H}), 7.71(\mathrm{dd}, J=1.8,0.8 \mathrm{~Hz}, 1 \mathrm{H}), 7.62$ (dd, $J=9.2,0.9 \mathrm{~Hz}, 1 \mathrm{H}), 7.39(\mathrm{~s}, 1 \mathrm{H}), 7.33(\mathrm{dd}, J=9.2,1.8 \mathrm{~Hz}, 1 \mathrm{H}), 7.17(\mathrm{~d}, J=8.4 \mathrm{~Hz}, 1 \mathrm{H})$, $7.14-7.07(\mathrm{~m}, 2 \mathrm{H}), 6.93-6.80(\mathrm{~m}, 2 \mathrm{H}), 6.68-6.58(\mathrm{~m}, 2 \mathrm{H}), 6.54(\mathrm{dd}, J=3.4,1.8 \mathrm{~Hz}, 1 \mathrm{H})$, $5.92(\mathrm{dd}, J=8.1,1.1 \mathrm{~Hz}, 1 \mathrm{H}), 5.17(\mathrm{t}, J=5.5 \mathrm{~Hz}, 1 \mathrm{H}), 4.35(\mathrm{~d}, J=5.4 \mathrm{~Hz}, 2 \mathrm{H}), 3.78(\mathrm{~s}, 3 \mathrm{H})$, $2.36(\mathrm{~s}, 3 \mathrm{H})$.

\section{Compound 3z}<smiles>CC(=O)Nc1cccc(-c2ccc3nc(-c4ccco4)c(Nc4ccccc4C)n3c2)c1</smiles>

${ }^{1} \mathrm{H}$ NMR $\left(400 \mathrm{MHz}, \mathrm{CDCl}_{3}\right) \delta 8.00(\mathrm{~s}, 1 \mathrm{H}), 7.84(\mathrm{~s}, 1 \mathrm{H}), 7.71-7.49(\mathrm{~m}, 3 \mathrm{H}), 7.47-7.38(\mathrm{~m}$, $2 \mathrm{H}), 7.33(\mathrm{t}, J=7.9 \mathrm{~Hz}, 1 \mathrm{H}), 7.24-7.11(\mathrm{~m}, 2 \mathrm{H}), 6.94(\mathrm{td}, J=7.8,1.6 \mathrm{~Hz}, 1 \mathrm{H}), 6.81(\mathrm{td}, J=$ $7.4,1.2 \mathrm{~Hz}, 1 \mathrm{H}), 6.70(\mathrm{~d}, J=3.4 \mathrm{~Hz}, 1 \mathrm{H}), 6.41(\mathrm{dd}, J=3.4,1.8 \mathrm{~Hz}, 1 \mathrm{H}), 6.10(\mathrm{dd}, J=8.0$, $1.2 \mathrm{~Hz}, 1 \mathrm{H}), 5.97(\mathrm{~s}, 1 \mathrm{H}), 2.50(\mathrm{~s}, 3 \mathrm{H}), 2.19(\mathrm{~s}, 3 \mathrm{H}) . \mathrm{MS}(\mathrm{ESI}+) \mathrm{m} / z: 423.2[\mathrm{M}+\mathrm{H}]^{+}$. 


\section{Compound 4b}<smiles>Cc1ccccc1Nc1c(-c2ccc[nH]2)nc2c(C)cccn12</smiles>

${ }^{1} \mathrm{H} \mathrm{NMR}\left(400 \mathrm{MHz}, \mathrm{CDCl}_{3}\right) \delta 9.77(\mathrm{~s}, 1 \mathrm{H}), 7.71(\mathrm{~d}, J=6.7 \mathrm{~Hz}, 1 \mathrm{H}), 7.20(\mathrm{~d}, J=7.3 \mathrm{~Hz}, 1 \mathrm{H})$, $7.02(\mathrm{~d}, J=7.0 \mathrm{~Hz}, 1 \mathrm{H}), 6.93(\mathrm{t}, J=7.5 \mathrm{~Hz}, 1 \mathrm{H}), 6.84-6.75(\mathrm{~m}, 2 \mathrm{H}), 6.71(\mathrm{t}, J=6.8 \mathrm{~Hz}$, $1 \mathrm{H}), 6.46-6.39(\mathrm{~m}, 1 \mathrm{H}), 6.25-6.09(\mathrm{~m}, 2 \mathrm{H}), 5.56(\mathrm{~s}, 1 \mathrm{H}), 2.64(\mathrm{~s}, 3 \mathrm{H}), 2.47(\mathrm{~s}, 3 \mathrm{H}) . \mathrm{MS}$ (ESI+) $m / z: 303.1[\mathrm{M}+\mathrm{H}]^{+}$.

\section{Compound 4d}<smiles>Cc1ccccc1Nc1c(C(C)c2ccccc2)nc2c(C)cccn12</smiles>

${ }^{1} \mathrm{H}$ NMR $\left(400 \mathrm{MHz}, \mathrm{CDCl}_{3}\right) \delta 7.44(\mathrm{~d}, J=6.8 \mathrm{~Hz}, 1 \mathrm{H}), 7.21(\mathrm{~d}, J=7.5 \mathrm{~Hz}, 2 \mathrm{H}), 7.09(\mathrm{dt}, J=$ 24.1, 7.2 Hz, 3H), 6.98 (d, $J=7.4 \mathrm{~Hz}, 1 \mathrm{H}), 6.85(\mathrm{~d}, J=6.8 \mathrm{~Hz}, 1 \mathrm{H}), 6.80$ (t, $J=7.8 \mathrm{~Hz}, 1 \mathrm{H})$, $6.63(\mathrm{t}, J=7.3 \mathrm{~Hz}, 1 \mathrm{H}), 6.50(\mathrm{t}, J=6.8 \mathrm{~Hz}, 1 \mathrm{H}), 5.82(\mathrm{~d}, J=8.1 \mathrm{~Hz}, 1 \mathrm{H}), 4.55(\mathrm{~s}, 1 \mathrm{H}), 4.36-$ $4.23(\mathrm{~m}, 1 \mathrm{H}), 2.58(\mathrm{~s}, 3 \mathrm{H}), 1.97(\mathrm{~s}, 3 \mathrm{H}), 1.62(\mathrm{~d}, J=7.3 \mathrm{~Hz}, 3 \mathrm{H}) . \mathrm{MS}(\mathrm{ESI}+) m / z: 342.2[\mathrm{M}+$ $\mathrm{H}]^{+}$.

\section{Compound 4e}<smiles>Cc1ccccc1Nc1c(C2C[C@H]3C=C[C@H]2C3)nc2c(C)cccn12</smiles>

${ }^{1} \mathrm{H}$ NMR $\left(400 \mathrm{MHz}, \mathrm{CDCl}_{3}\right) \delta 7.49(\mathrm{~d}, J=6.7 \mathrm{~Hz}, 1 \mathrm{H}), 7.19-7.12(\mathrm{~m}, 1 \mathrm{H}), 6.96-6.88(\mathrm{~m}$, 2H), $6.75(\mathrm{td}, J=7.4,1.2 \mathrm{~Hz}, 1 \mathrm{H}), 6.59(\mathrm{t}, J=6.8 \mathrm{~Hz}, 1 \mathrm{H}), 6.27(\mathrm{dd}, J=5.8,3.1 \mathrm{~Hz}, 1 \mathrm{H})$, $5.95(\mathrm{dd}, J=5.7,2.9 \mathrm{~Hz}, 1 \mathrm{H}), 5.90(\mathrm{dd}, J=8.1,1.2 \mathrm{~Hz}, 1 \mathrm{H}), 5.41(\mathrm{~s}, 1 \mathrm{H}), 3.63(\mathrm{dt}, J=9.0$, $4.2 \mathrm{~Hz}, 1 \mathrm{H}), 3.35(\mathrm{dq}, J=3.7,1.8 \mathrm{~Hz}, 1 \mathrm{H}), 2.92(\mathrm{t}, J=3.0 \mathrm{~Hz}, 1 \mathrm{H}), 2.63(\mathrm{~s}, 3 \mathrm{H}), 2.37$ (s, $3 \mathrm{H}), 2.13-2.02(\mathrm{~m}, 1 \mathrm{H}), 1.63(\mathrm{ddd}, J=11.8,4.8,2.5 \mathrm{~Hz}, 1 \mathrm{H}), 1.51-1.39$ (m, 2H). MS (ESI+) $m / z: 330.2[\mathrm{M}+\mathrm{H}]^{+}$. 


\section{Compound 4f}<smiles>Cc1ccccc1Nc1c(C2CCC2)nc2c(C)cccn12</smiles>

${ }^{1} \mathrm{H}$ NMR $\left(400 \mathrm{MHz}, \mathrm{CDCl}_{3}\right) \delta 7.64(\mathrm{~d}, J=6.7 \mathrm{~Hz}, 1 \mathrm{H}), 7.16(\mathrm{~d}, J=6.9 \mathrm{~Hz}, 1 \mathrm{H}), 7.03-6.90$ $(\mathrm{m}, 2 \mathrm{H}), 6.77(\mathrm{t}, J=7.4 \mathrm{~Hz}, 1 \mathrm{H}), 6.71-6.63(\mathrm{~m}, 1 \mathrm{H}), 6.04(\mathrm{~d}, J=8.0 \mathrm{~Hz}, 1 \mathrm{H}), 5.21(\mathrm{~s}, 1 \mathrm{H})$, $3.71(\mathrm{p}, J=8.9 \mathrm{~Hz}, 1 \mathrm{H}), 2.69(\mathrm{~d}, J=2.7 \mathrm{~Hz}, 3 \mathrm{H}), 2.58-2.45(\mathrm{~m}, 2 \mathrm{H}), 2.40(\mathrm{~s}, 3 \mathrm{H}), 2.33-$ $2.21(\mathrm{~m}, 2 \mathrm{H}), 2.06-1.82(\mathrm{~m}, 2 \mathrm{H})$. MS (ESI+) $m / z: 292.1[\mathrm{M}+\mathrm{H}]^{+}$.

\section{Compound 5d}<smiles>Cc1cccc(C)c1Nc1c(-c2ccco2)nc2c(C)cccn12</smiles>

${ }^{1} \mathrm{H}$ NMR $\left(400 \mathrm{MHz}, \mathrm{CDCl}_{3}\right) \delta 7.57(\mathrm{~d}, J=6.9 \mathrm{~Hz}, 1 \mathrm{H}), 7.47(\mathrm{~d}, J=9.3 \mathrm{~Hz}, 1 \mathrm{H}), 7.18-6.93$ $(\mathrm{m}, 3 \mathrm{H}), 6.93-6.79(\mathrm{~m}, 1 \mathrm{H}), 6.77-6.60(\mathrm{~m}, 2 \mathrm{H}), 6.42(\mathrm{~s}, 1 \mathrm{H}), 5.45(\mathrm{~s}, 1 \mathrm{H}), 2.97-2.58(\mathrm{~m}$, $3 \mathrm{H}), 2.03(\mathrm{~d}, J=11.0 \mathrm{~Hz}, 6 \mathrm{H})$.

\section{Compound 5e}<smiles>Cc1cccn2c(NC3CCCCC3)c(-c3ccco3)nc12</smiles>

${ }^{1} \mathrm{H}$ NMR $\left(400 \mathrm{MHz}, \mathrm{CDCl}_{3}\right) \delta 7.92(\mathrm{~d}, J=6.9 \mathrm{~Hz}, 1 \mathrm{H}), 7.49(\mathrm{~s}, 1 \mathrm{H}), 6.88(\mathrm{dd}, J=16.9,5.0$ $\mathrm{Hz}, 2 \mathrm{H}), 6.67(\mathrm{t}, J=6.8 \mathrm{~Hz}, 1 \mathrm{H}), 6.51(\mathrm{~s}, 1 \mathrm{H}), 3.55(\mathrm{~d}, J=6.9 \mathrm{~Hz}, 1 \mathrm{H}), 3.09-2.85(\mathrm{~m}, 1 \mathrm{H})$, $2.60(\mathrm{~s}, 3 \mathrm{H}), 1.96-1.50(\mathrm{~m}, 4 \mathrm{H}), 1.22(\mathrm{dt}, J=21.1,10.7 \mathrm{~Hz}, 6 \mathrm{H})$. MS (ESI+) $m / z: 296.1$ [M $+\mathrm{H}]^{+}$.

\section{Compound $5 f$}<smiles>Cc1cccn2c(NC(C)(C)C)c(-c3ccco3)nc12</smiles> 
${ }^{1} \mathrm{H}$ NMR $\left(600 \mathrm{MHz}, \mathrm{CDCl}_{3}\right) \delta 8.10-8.06(\mathrm{~m}, 1 \mathrm{H}), 7.46(\mathrm{td}, J=1.9,1.0 \mathrm{~Hz}, 1 \mathrm{H}), 6.90-6.86$ $(\mathrm{m}, 2 \mathrm{H}), 6.65-6.59(\mathrm{~m}, 1 \mathrm{H}), 6.49(\mathrm{dt}, J=3.3,1.6 \mathrm{~Hz}, 1 \mathrm{H}), 3.46(\mathrm{~s}, 1 \mathrm{H}), 2.59(\mathrm{dd}, J=2.1$, $1.1 \mathrm{~Hz}, 3 \mathrm{H}), 1.11(\mathrm{~s}, 9 \mathrm{H}) . \mathrm{MS}(\mathrm{ESI}+) \mathrm{m} / z: 269.1[\mathrm{M}+\mathrm{H}]^{+}$.

\section{Compound 6c}<smiles>Cc1ccccc1Nc1c(C2CC3C=CC2C3)nc2c(C)cccn12</smiles>

${ }^{1} \mathrm{H}$ NMR $\left(400 \mathrm{MHz}, \mathrm{CDCl}_{3}\right) \delta 7.83(\mathrm{~d}, J=6.8 \mathrm{~Hz}, 1 \mathrm{H}), 6.97(\mathrm{~d}, J=7.5 \mathrm{~Hz}, 2 \mathrm{H}), 6.89(\mathrm{dt}, J=$ $6.8,1.2 \mathrm{~Hz}, 1 \mathrm{H}), 6.81(\mathrm{t}, J=7.5 \mathrm{~Hz}, 1 \mathrm{H}), 6.68(\mathrm{t}, J=6.8 \mathrm{~Hz}, 1 \mathrm{H}), 6.04(\mathrm{dd}, J=5.7,2.9 \mathrm{~Hz}$, $1 \mathrm{H}), 5.86(\mathrm{dd}, J=5.7,3.0 \mathrm{~Hz}, 1 \mathrm{H}), 4.99(\mathrm{~s}, 1 \mathrm{H}), 2.94(\mathrm{~s}, 1 \mathrm{H}), 2.62-2.60(\mathrm{~m}, 1 \mathrm{H}), 2.59(\mathrm{~s}$, $3 \mathrm{H}), 2.43$ (ddd, $J=8.8,4.6,1.5 \mathrm{~Hz}, 1 \mathrm{H}), 2.21-2.12(\mathrm{~m}, 1 \mathrm{H}), 2.10-2.05(\mathrm{~m}, 1 \mathrm{H}), 1.98$ (s, $6 \mathrm{H}), 1.36-1.21(\mathrm{~m}, 2 \mathrm{H})$. MS (ESI+) $m / z: 344.2[\mathrm{M}+\mathrm{H}]^{+}$.

\section{Compound 8a}<smiles>Cc1cc(Cl)cn2c(NC3CCCCC3)c(-c3ccco3)nc12</smiles>

${ }^{1} \mathrm{H}$ NMR $\left(400 \mathrm{MHz}, \mathrm{CDCl}_{3}\right) \delta 7.97(\mathrm{~s}, 1 \mathrm{H}), 7.51(\mathrm{dd}, J=1.9,0.8 \mathrm{~Hz}, 1 \mathrm{H}), 7.04-6.79(\mathrm{~m}$, $2 \mathrm{H}), 6.53(\mathrm{dd}, J=3.4,1.8 \mathrm{~Hz}, 1 \mathrm{H}), 3.56(\mathrm{~d}, J=7.0 \mathrm{~Hz}, 1 \mathrm{H}), 2.94$ (dtd, $J=10.4,6.8,3.5 \mathrm{~Hz}$, $1 \mathrm{H}), 2.62(\mathrm{~s}, 3 \mathrm{H}), 1.95-1.81(\mathrm{~m}, 2 \mathrm{H}), 1.81-1.67(\mathrm{~m}, 2 \mathrm{H}), 1.38-1.04(\mathrm{~m}, 6 \mathrm{H})$. MS (ESI+) $m / z: 330.1\left[\mathrm{M}+\mathrm{H},{ }^{35} \mathrm{Cl}\right]^{+}, 332.1\left[\mathrm{M}+\mathrm{H},{ }^{37} \mathrm{Cl}\right]^{+}$.

\section{Compound 8b}<smiles>Cc1cc(Cl)cn2c(NC(C)(C)C)c(-c3ccco3)nc12</smiles>

${ }^{1} \mathrm{H}$ NMR $\left(400 \mathrm{MHz}, \mathrm{CDCl}_{3}\right) \delta 8.14(\mathrm{dd}, J=2.0,0.8 \mathrm{~Hz}, 1 \mathrm{H}), 7.49(\mathrm{dd}, J=1.8,0.8 \mathrm{~Hz}, 1 \mathrm{H})$, $6.89(\mathrm{ddd}, J=9.2,2.8,0.9 \mathrm{~Hz}, 2 \mathrm{H}), 6.52(\mathrm{dd}, J=3.4,1.8 \mathrm{~Hz}, 1 \mathrm{H}), 3.48(\mathrm{~s}, 1 \mathrm{H}), 2.60$ (t, $J=$ $1.0 \mathrm{~Hz}, 3 \mathrm{H}), 1.13(\mathrm{~s}, 9 \mathrm{H})$. MS (ESI+) $m / z: 304.1\left[\mathrm{M}+\mathrm{H},{ }^{35} \mathrm{Cl}\right]^{+}, 306.0\left[\mathrm{M}+\mathrm{H},{ }^{37} \mathrm{Cl}\right]^{+}$.

\section{Compound 8c}


<smiles>N#Cc1cccn2c(NC3CCCCC3)c(-c3ccco3)nc12</smiles>

${ }^{1} \mathrm{H}$ NMR $\left(400 \mathrm{MHz}, \mathrm{CDCl}_{3}\right) \delta 8.25(\mathrm{~d}, J=7.5 \mathrm{~Hz}, 1 \mathrm{H}), 7.59-7.50(\mathrm{~m}, 2 \mathrm{H}), 7.02(\mathrm{~d}, J=3.5$ $\mathrm{Hz}, 1 \mathrm{H}), 6.89-6.79(\mathrm{~m}, 1 \mathrm{H}), 6.60-6.52(\mathrm{~m}, 1 \mathrm{H}), 3.61(\mathrm{~s}, 1 \mathrm{H}), 3.02-2.89(\mathrm{~m}, 1 \mathrm{H}), 1.92-$ $1.83(\mathrm{~m}, 2 \mathrm{H}), 1.79-1.71(\mathrm{~m}, 2 \mathrm{H}), 1.36-1.14(\mathrm{~m}, 6 \mathrm{H}) . \mathrm{MS}(\mathrm{ESI}+) \mathrm{m} / z: 307.1[\mathrm{M}+\mathrm{H}]^{+}$.

\section{Compound 8d}<smiles>CC(C)(C)Nc1c(-c2ccco2)nc2c(C#N)cccn12</smiles>

${ }^{1} \mathrm{H}$ NMR $\left(400 \mathrm{MHz}, \mathrm{CDCl}_{3}\right) \delta 8.44(\mathrm{dd}, J=7.0,1.2 \mathrm{~Hz}, 1 \mathrm{H}), 7.56(\mathrm{dd}, J=7.0,1.2 \mathrm{~Hz}, 1 \mathrm{H})$, $7.51(\mathrm{dd}, J=1.8,0.8 \mathrm{~Hz}, 1 \mathrm{H}), 7.03(\mathrm{dd}, J=3.4,0.8 \mathrm{~Hz}, 1 \mathrm{H}), 6.82(\mathrm{t}, J=7.0 \mathrm{~Hz}, 1 \mathrm{H}), 6.55$ (dd, $J=3.4,1.8 \mathrm{~Hz}, 1 \mathrm{H}), 3.59$ (s, 1H), $1.15(\mathrm{~s}, 9 \mathrm{H})$. MS (ESI+) $m / z: 281.1[\mathrm{M}+\mathrm{H}]^{+}$.

\section{Compound 8e}<smiles>FC(F)(F)c1cccn2c(NC3CCCCC3)c(-c3ccco3)nc12</smiles>

${ }^{1} \mathrm{H} \mathrm{NMR}\left(600 \mathrm{MHz}, \mathrm{CDCl}_{3}\right) \delta 8.23(\mathrm{~d}, J=6.8 \mathrm{~Hz}, 1 \mathrm{H}), 7.51(\mathrm{dd}, J=1.8,0.8 \mathrm{~Hz}, 1 \mathrm{H}), 7.48$ $(\mathrm{d}, J=7.1 \mathrm{~Hz}, 1 \mathrm{H}), 7.02(\mathrm{~d}, J=3.3 \mathrm{~Hz}, 1 \mathrm{H}), 6.84(\mathrm{t}, J=6.9 \mathrm{~Hz}, 1 \mathrm{H}), 6.53(\mathrm{dd}, J=3.4,1.8$ $\mathrm{Hz}, 1 \mathrm{H}), 2.94(\mathrm{tt}, J=10.6,3.8 \mathrm{~Hz}, 1 \mathrm{H}), 1.92-1.84(\mathrm{~m}, 2 \mathrm{H}), 1.74(\mathrm{dq}, J=7.6,4.1,3.4 \mathrm{~Hz}$, $2 \mathrm{H}), 1.34-1.13(\mathrm{~m}, 6 \mathrm{H})$. MS (ESI+) $m / z: 350.1[\mathrm{M}+\mathrm{H}]^{+}$.

\section{Compound 10a}<smiles>Cc1ccccc1Nc1c(-c2ccco2)nc2ncccn12</smiles>

${ }^{1} \mathrm{H}$ NMR $\left(400 \mathrm{MHz}, \mathrm{CDCl}_{3}\right) \delta 8.54(\mathrm{dd}, J=4.1,2.1 \mathrm{~Hz}, 1 \mathrm{H}), 8.02(\mathrm{dd}, J=6.8,2.0 \mathrm{~Hz}, 1 \mathrm{H})$, $7.44(\mathrm{dd}, J=1.8,0.8 \mathrm{~Hz}, 1 \mathrm{H}), 7.21(\mathrm{~d}, J=6.8 \mathrm{~Hz}, 1 \mathrm{H}), 7.01-6.88(\mathrm{~m}, 2 \mathrm{H}), 6.88-6.78(\mathrm{~m}$, 
2H), $6.46(\mathrm{dd}, J=3.4,1.8 \mathrm{~Hz}, 1 \mathrm{H}), 6.05(\mathrm{~d}, J=8.0 \mathrm{~Hz}, 1 \mathrm{H}), 5.82(\mathrm{~s}, 1 \mathrm{H}), 2.47(\mathrm{~s}, 3 \mathrm{H}) . \mathrm{MS}$ $(\mathrm{ESI}+) \mathrm{m} / z: 291.1[\mathrm{M}+\mathrm{H}]^{+}$.

\section{Compound 10b}<smiles>Cc1ccccc1Nc1c(-c2ccco2)nc2ncc(Br)cn12</smiles>

${ }^{1} \mathrm{H}$ NMR $\left(400 \mathrm{MHz}, \mathrm{CDCl}_{3}\right) \delta 8.52(\mathrm{~d}, J=2.4 \mathrm{~Hz}, 1 \mathrm{H}), 8.15(\mathrm{~d}, J=2.5 \mathrm{~Hz}, 1 \mathrm{H}), 7.46(\mathrm{~d}, J=$ $1.7 \mathrm{~Hz}, 1 \mathrm{H}), 7.24(\mathrm{~d}, J=7.1 \mathrm{~Hz}, 1 \mathrm{H}), 7.01(\mathrm{t}, J=8.0 \mathrm{~Hz}, 1 \mathrm{H}), 6.95(\mathrm{~d}, J=3.4 \mathrm{~Hz}, 1 \mathrm{H}), 6.87$ (t, $J=7.4 \mathrm{~Hz}, 1 \mathrm{H}), 6.48(\mathrm{dd}, J=3.5,1.8 \mathrm{~Hz}, 1 \mathrm{H}), 6.07$ (d, $J=7.9 \mathrm{~Hz}, 1 \mathrm{H}), 5.82(\mathrm{~s}, 1 \mathrm{H}), 2.47$ (s, 3H). MS (ESI+) $m / z: 369.1\left[\mathrm{M}+\mathrm{H},{ }^{79} \mathrm{Br}\right]^{+}, 371.0\left[\mathrm{M}+\mathrm{H},{ }^{81} \mathrm{Br}\right]^{+}$.

\section{Compound 10c}<smiles>Cc1ccccc1Nc1c(-c2ccco2)nc2cnccn12</smiles>

${ }^{1} \mathrm{H}$ NMR (400 MHz, $\left.\mathrm{CDCl}_{3}\right) \delta 9.07(\mathrm{~d}, J=1.4 \mathrm{~Hz}, 1 \mathrm{H}), 7.84(\mathrm{~d}, J=4.6 \mathrm{~Hz}, 1 \mathrm{H}), 7.65(\mathrm{dd}, J$ $=4.5,1.5 \mathrm{~Hz}, 1 \mathrm{H}), 7.49(\mathrm{~d}, J=1.7 \mathrm{~Hz}, 1 \mathrm{H}), 7.24(\mathrm{~d}, J=13.5 \mathrm{~Hz}, 1 \mathrm{H}), 6.99$ (t, $J=7.7 \mathrm{~Hz}$, $1 \mathrm{H}), 6.91-6.82(\mathrm{~m}, 1 \mathrm{H}), 6.49(\mathrm{dd}, J=3.4,1.8 \mathrm{~Hz}, 1 \mathrm{H}), 6.04(\mathrm{~d}, J=8.1 \mathrm{~Hz}, 1 \mathrm{H}), 5.82(\mathrm{~s}$, 1H), $2.47(\mathrm{~s}, 3 \mathrm{H})$. MS (ESI+) $\mathrm{m} / z: 291.1[\mathrm{M}+\mathrm{H}]^{+}$.

\section{Compound 10d}<smiles>Cc1ccccc1Nc1c(-c2ccco2)nc2ccncn12</smiles>

${ }^{1} \mathrm{H}$ NMR (400 MHz, $\left.\mathrm{CDCl}_{3}\right) \delta 8.65(\mathrm{~d}, J=1.5 \mathrm{~Hz}, 1 \mathrm{H}), 7.97(\mathrm{~d}, J=6.5 \mathrm{~Hz}, 1 \mathrm{H}), 7.51(\mathrm{~d}, J=$ $1.5 \mathrm{~Hz}, 1 \mathrm{H}), 7.49(\mathrm{td}, J=1.9,1.1 \mathrm{~Hz}, 1 \mathrm{H}), 7.23(\mathrm{~d}, J=7.2 \mathrm{~Hz}, 1 \mathrm{H}), 7.00(\mathrm{td}, J=7.7,1.5 \mathrm{~Hz}$, $1 \mathrm{H}), 6.86(\mathrm{td}, J=7.4,1.2 \mathrm{~Hz}, 1 \mathrm{H}), 6.82(\mathrm{dd}, J=3.4,0.8 \mathrm{~Hz}, 1 \mathrm{H}), 6.48(\mathrm{dd}, J=3.4,1.8 \mathrm{~Hz}$, $1 \mathrm{H}), 6.17(\mathrm{dd}, J=8.0,1.1 \mathrm{~Hz}, 1 \mathrm{H}), 5.70(\mathrm{~s}, 1 \mathrm{H}), 2.47(\mathrm{~s}, 3 \mathrm{H})$.

\section{Compound 10e}

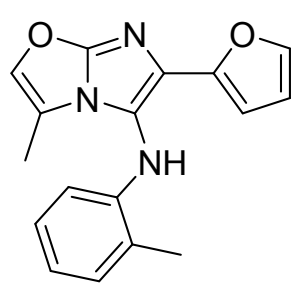


${ }^{1} \mathrm{H}$ NMR $\left(400 \mathrm{MHz}, \mathrm{CDCl}_{3}\right) \delta 8.54(\mathrm{~d}, J=11.3 \mathrm{~Hz}, 1 \mathrm{H}), 8.50-8.38(\mathrm{~m}, 1 \mathrm{H}), 7.98-7.85(\mathrm{~m}$, 1H), 7.77 (s, 1H), $7.42-6.94(\mathrm{~m}, 5 \mathrm{H}), 2.30(\mathrm{~s}, 3 \mathrm{H}), 2.28$ (s, 3H).

\section{Compound 10f}

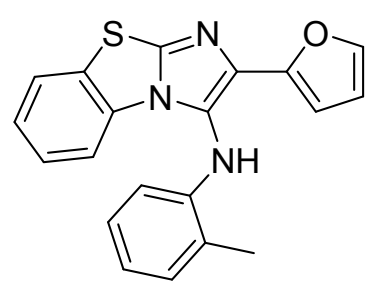

${ }^{1} \mathrm{H}$ NMR $\left(400 \mathrm{MHz}, \mathrm{CDCl}_{3}\right) \delta 7.71-7.61(\mathrm{~m}, 1 \mathrm{H}), 7.55-7.43(\mathrm{~m}, 1 \mathrm{H}), 7.37(\mathrm{dd}, J=1.8$, $0.8 \mathrm{~Hz}, 1 \mathrm{H}), 7.34-7.18(\mathrm{~m}, 2 \mathrm{H}), 7.01-6.91(\mathrm{~m}, 1 \mathrm{H}), 6.88-6.78(\mathrm{~m}, 1 \mathrm{H}), 6.63(\mathrm{~d}, J=3.4$ $\mathrm{Hz}, 1 \mathrm{H}), 6.46-6.32(\mathrm{~m}, 2 \mathrm{H}), 5.86(\mathrm{~s}, 1 \mathrm{H}), 2.50(\mathrm{~s}, 3 \mathrm{H}) . \mathrm{MS}(\mathrm{ESI}+) \mathrm{m} / z: 346.1[\mathrm{M}+\mathrm{H}]^{+}$.

\section{Compound 10g}

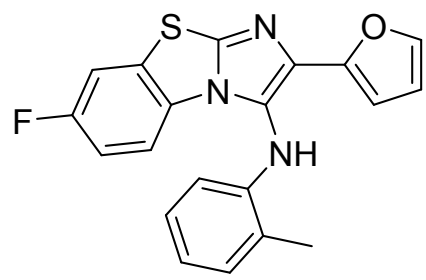

${ }^{1} \mathrm{H}$ NMR $\left(600 \mathrm{MHz}, \mathrm{CDCl}_{3}\right) \delta 7.43(\mathrm{dd}, J=9.0,4.5 \mathrm{~Hz}, 1 \mathrm{H}), 7.39(\mathrm{dd}, J=7.9,2.5 \mathrm{~Hz}, 1 \mathrm{H})$, $7.36(\mathrm{dd}, J=1.8,0.8 \mathrm{~Hz}, 1 \mathrm{H}), 7.25(\mathrm{~d}, J=7.3 \mathrm{~Hz}, 1 \mathrm{H}), 7.04-6.99(\mathrm{~m}, 1 \mathrm{H}), 7.00-6.93$ (m, $1 \mathrm{H}), 6.85(\mathrm{td}, J=7.4,1.2 \mathrm{~Hz}, 1 \mathrm{H}), 6.66(\mathrm{~d}, J=3.7 \mathrm{~Hz}, 1 \mathrm{H}), 6.40(\mathrm{dd}, J=3.4,1.8 \mathrm{~Hz}, 1 \mathrm{H})$, $6.35(\mathrm{~d}, J=8.1 \mathrm{~Hz}, 1 \mathrm{H}), 6.01(\mathrm{~s}, 1 \mathrm{H}), 2.51(\mathrm{~s}, 3 \mathrm{H}) . \mathrm{MS}(\mathrm{ESI}+) \mathrm{m} / z: 364.1[\mathrm{M}+\mathrm{H}]^{+}$. 